\title{
In Situ Tracking of Picoscale Atomic Displacements with Millisecond Temporal- Resolution During Exchange and Diffusion Processes in Energy Materials in TEM
}

\author{
Barnaby D.A. Levin ${ }^{1 *}$, Ethan L. Lawrence ${ }^{1}$, Piyush Haluai ${ }^{1}$, and Peter A. Crozier ${ }^{1}$ \\ 1. School for Engineering of Matter, Transport, and Energy, Arizona State University, Arizona, USA. \\ * Corresponding author: blevin2@asu.edu
}

Advances in detector technology for transmission electron microscopy (TEM) [1] have made it possible to acquire image series of TEM specimens with atomic scale spatial resolution and a temporal resolution on the order of milliseconds. These advances raise the possibility of measuring dynamic changes in atomic structure, which may yield important insights into fundamental physical processes in materials, such as atomic diffusion and oxygen exchange. This has driven efforts to develop software to accurately track the changes in the position of atomic columns in TEM image series, primarily using centroid fitting methods [2].

Here, we present a new approach to track changes in both the position and intensity of atomic columns using a two-dimensional elliptical gaussian fitting procedure. An example fit to experimental data is shown in Figure 1. The co-ordinates of the maximum of the 2D Gaussian can be used to define the position of the atomic column, and the intensity of the column may be estimated by integrating the 2D Gaussian. Iteratively fitting 2D Gaussians to atomic columns in each frame of an image series allows us to determine changes in the positions of atomic columns to picoscale accuracy and track variations in atomic column intensity, which can then be related to dynamic structural changes.

We have applied our 2D Gaussian fitting approach to analyze images of $\mathrm{CeO}_{2}$ nanoparticles, in which surface cerium cation columns are observed to undergo small displacements between individual frames. Comparison of experimental images with simulations has indicated that cerium column displacements of $10 \mathrm{pm}$ or more are caused by the creation and annihilation of oxygen vacancies adjacent to the cerium columns $[3,4]$. During an image series, the most active surface sites for oxygen vacancy creation are therefore expected to undergo a greater number of displacements. Figure 2 shows a summed image of a stepped (111) $\mathrm{CeO}_{2}$ nanoparticle surface from a 10 frame image series. Comparison of the intensity of the atomic columns with multislice simulations (not shown) suggests that there are between 1 and 6 atoms per column at each surface site. Colored circles overlaid on the image indicate the frequency of cerium column displacements greater than $10 \mathrm{pm}$ during the image series. The highest displacement frequencies are observed at step edges, and on the strained terrace in the center of the image, suggesting that these atomic sites have a higher activity for oxygen vacancy creation and annihilation.

We are currently applying our 2D Gaussian fitting approach to characterize a number of other datasets, with temporal resolutions of up to $2.5 \mathrm{~ms}$ per frame. We are exploring how noise imposes limitations on calculating atomic column positions and intensities [5].

\section{References:}

[1] G McMullan et. al., Ultramicroscopy 147 (2014), p. 156.

[2] Z Hussaini et. al., Ultramicroscopy 186 (2018) p. 139.

[3] EL Lawrence et. al., Microscopy and Microanalysis 24(S1) (2018), p. 54. 
[4] T Boland et. al., Microscopy and Microanalysis 24(S1) (2018), p. 144.

[5] We gratefully acknowledge support from NSF DMR-1308085 and ASU's John M. Cowley Center for High Resolution Electron Microscopy.

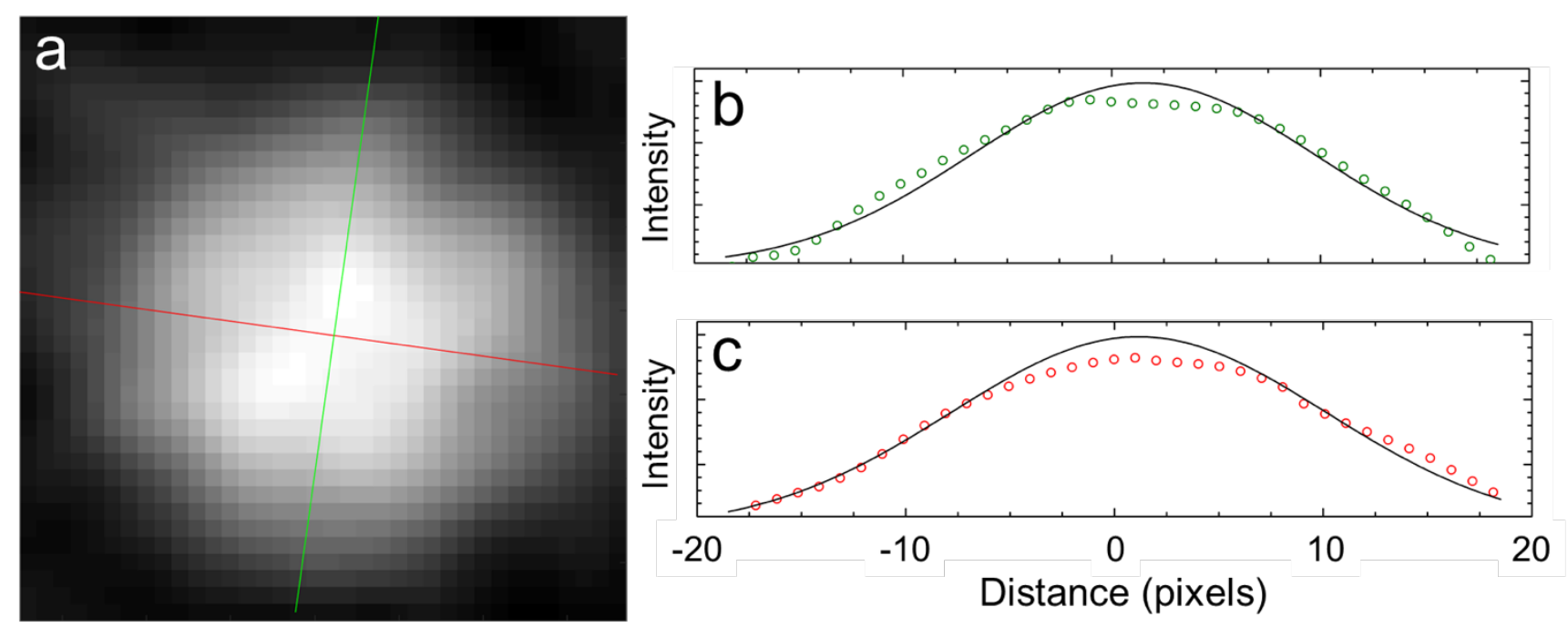

Figure 1. a TEM image of a single cerium atomic column at the surface of a $\mathrm{CeO}_{2}$ particle. The width of the image is approximately $140 \mathrm{pm}$. The image was acquired with negative spherical aberration, and therefore shows bright atom contrast. The red and green lines across the image show the major and minor axes of a 2D elliptical Gaussian fit to the atomic column. b A cross section through the image along the green line shown in (a). Green circles represent experimental data points, and the black line is a cross section of the 2D Gaussian fit. c A cross section through the image along the red line shown in (a). Red circles represent experimental data points, and the black line is a cross section of the 2D Gaussian fit.

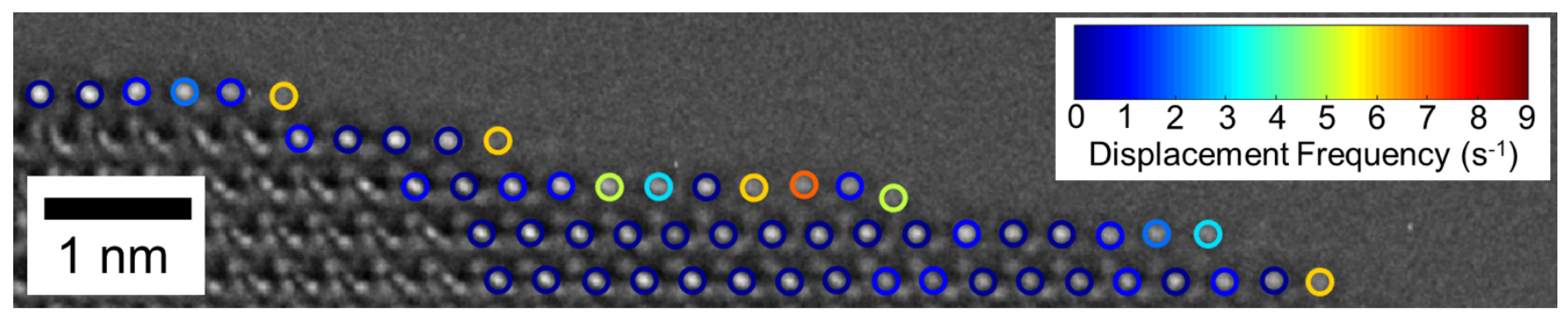

Figure 2. Experimental image of the stepped (111) surface of a $\mathrm{CeO}_{2}$ nanoparticle. The image is the sum of a series of 10 frames, acquired at $100 \mathrm{~ms}$ per frame. The colored circles overlaid onto each cerium atomic column indicate the number of times the column is displaced by more than $10 \mathrm{pm}$ from its previous position (the displacement frequency). The highest displacement frequencies are observed at step edge and strained sites, which suggests that they are the most active sites on the (111) surface for oxygen vacancy creation and annihilation. 